\title{
Estimating Inert Gas Bubbling from Simple SCUBA Diving Parameters
}

\section{(C) $(9)$}

Authors

Andreas Fichtner ${ }^{1,2}$, Benedikt Brunner ${ }^{3}$, Thomas Pohl ${ }^{4}$, Thomas Grab ${ }^{4}$, Tobias Fieback ${ }^{4}$, Thea Koch ${ }^{1}$

\section{Affiliations}

1 Department of Anesthesiology and Intensive Care Medicine, University Hospital Carl Gustav Carus, Dresden, Germany

2 Emergency Department, Kreiskrankenhaus Freiberg $\mathrm{gGmbH}$, Freiberg, Germany

3 Faculty of Science and Technology, University of Algarve, Faro, Portugal

4 Scientific Diving Center, TU Bergakademie Freiberg University, Freiberg, Germany

\section{Key words}

decompression, bubble grade, SCUBA diving, Doppler

ultrasound, risk management

accepted 14.12.2020

published online 27.01 .2021

Bibliography

Int J Sports Med 2021; 42: 840-846

DOI $10.1055 / \mathrm{a}-1342-8030$

ISSN $0172-4622$

(c) 2021. The Author(s).

This is an open access article published by Thieme under the terms of the Creative Commons Attribution-NonDerivative-NonCommercial-License, permitting copying and reproduction so long as the original work is given appropriate credit. Contents may not be used for commercial purposes, or adapted, remixed, transformed or built upon. (https://creativecommons.org/ licenses/by-nc-nd/4.0/)

Georg Thieme Verlag KG, Rüdigerstraße 14,

70469 Stuttgart, Germany

\section{Correspondence}

Andreas Fichtner

Department of Anesthesiology and Intensive Care Medicine

University Hospital Carl Gustav Carus

Technische Universität Dresden

01307 Dresden

Germany

Tel.: +49-3731-772198

andreas.fichtner@kkh-freiberg.de

\begin{abstract}
Inert gas bubbles frequently occur in SCUBA divers' vascular systems, eventually leading to decompression accidents. Only in professional settings, dive profiles can be adjusted on individual basis depending on bubble grades detected through ultrasonography. A total of 342 open-circuit air dives following sports diving profiles were assessed using echocardiography. Subsequently, (Eftedal-Brubakk) bubble grades were correlated with dive and individual parameters. Post-dive cardiac bubbles were observed in $47 \%$ of all dives and bubble grades were significantly correlated with depth $(r=0.46)$, air consumption $(r=0.41)$, age $(r=0.25)$, dive time $(r=0.23)$, decompression diving $(r=0.19)$, surface time $(r=-0.12)$. Eftedal-Brubakk categorical bubble grades for sports diving with compressed air can be approximated by bubble grade $=\left(\right.$ age $* 50^{-1}-$ surface time $* 150^{-1}+$ maximum depth * $45^{-1}+$ air consumption * 4500-1 $)^{2}$ (units in years, hours, meter, and bar * liter; $R^{2}=0.31$ ). Thus, simple dive and individual parameters allow reasonable estimation of especially relevant medium to higher bubble grades for information on relevant decompression stress after ascent. Echo bubble grade 0 is overestimated by the formula derived. However, echo might fail to detect minor bubbling only. The categorical prediction of individual decompression stress with simple bio and dive data should be evaluated further to be developed towards dive computer included automatic ex-post information for decisionmaking on individual safety measures.
\end{abstract}




\section{Introduction}

Inert gas bubbles are known to cause decompression sickness in self-contained underwater breathing apparatus (SCUBA) diving accidents and occur after inert gas (specifically Nitrogen in compressed air diving) supersaturation and omitted decompression brakes and fast ascents. Inert gas saturation is not only a function of dive time, depth, and ascent speed, but also depending on individual factors like dehydration, stress, age and others [1,2]. Mainly without any symptoms, inert gas bubbles frequently occur in SCUBA divers after ascending from a dive, and the number of bubbles finally determines a symptomatic decompression incident. However, there are divers that are more prone to post-dive bubbling compared to others after the same inert gas exposure without differences in oxidative stress or antioxidant capacity [3]. A high amount of detectable bubbles in dives within normal sports diving limits is related to symptoms of decompression sickness in around $10 \%$ and even higher in mixed gas commercial diving [4-6].

Said dive parameters, as well as personal health condition and exertion during the dive [7], are contributing to bubble formation and eventually to a decompression incident. In order to avoid such, modern watch-like dive computers provide restrictive safety margins and real-time calculations of saturation and desaturation of many virtual tissue speeds during a dive but cannot entirely avoid diving accidents. Furthermore, the majority of diving accidents caused by decompression sickness is not predicted by the adopted decompression algorithm [7].

Bubble occurrence after a dive is not stable, can be provoked by physical activity, and the typical bubble peak is $30-45$ min after a dive $[1,7]$. Echocardiographic bubble grading as the current gold standard is non-linear, and the categorical grading somewhat obscures the potential of high diagnostic accuracy with modern ultrasound technology according to current guidelines [8]. However, since bubble occurrence is frequent and does not equal a diving accident, the diagnostic information of just medium to higher bubble grades seem to be relevant to decide on behavioral adjustments after diving.

Our aim is to find a relationship between dive profile and individual characteristics and the severity of bubble occurrence after a dive, in order to allow sports divers to estimate decompression stress as a precondition to observe appropriate safety measures, e. g., increased surface intervals and fluid intake. With this new approach, we aim to close the gap in the mainly lacking post-dive ultrasound bubble assessment to quantify the inter- and intraindividually variable decompression response and to finally help avoid diving accidents.

\section{Materials and Methods}

We examined 41 scuba divers of different ages, gender and body characteristics in a total of 342 single and repetitive open-circuit compressed air dives within sports diving limits using wet-suit and modern real-time dive computers in shallow and deep, fresh and salt water. Dives were standard educational sports dives for research divers with underwater tasks like orientation, buoy operation, measurements, but without any heavy exercise, current or workload. Dive computer limits such as ascent speed and decompression breaks were observed and monitored by analyzing the log of the dive computers. Divers and dive profiles covered a broad spectrum and were not standardized. In contrast, post-dive physical behavior and bubble recording were standardized: All divers were assessed for weight (empty bladder) including bio-impedance estimated percentages of body fat, water and muscle content (Beurer BF 105 diagnostic scale), height, diseases, vital signs and activity level before and after any dive. Daily fluid intake was recorded throughout the whole study period for each individual in $100 \mathrm{ml}$-intervals. Surface intervals were recorded before any dive and specified in hours up to a maximum of $48 \mathrm{~h}$. After dive ascent, all divers walked back to the dive base with full equipment (approx. 100 meters, provocation period), dressed off and reported directly after the dive (30 min, the time interval was recorded) for postdive assessment without any rest, eating or drinking during this period. Dive parameters (depth, time, total air consumption, safety and decompression stops, pre-dive surface intervals up to $24 \mathrm{~h}$ ), including impaired well-being during the dive due to stress, cold, equalization problems, and others were recorded while standing. Total air consumption was calculated using tank pressure difference (pre- and post-dive) and tank size. After urinating, body weight and impedance-derived percentages of body compositions were measured, and guided Doppler Self-Monitoring for bubble detection was performed. Forty minutes after the dives, standardized echocardiography to record inert gas bubbles was performed in laying supine position at subcostal and apical approach using a GE Logic e (GE Healthcare, Solingen) ultrasound machine with a curved array multi-frequency probe. After signal optimization and visual bubble detection within $1 \mathrm{~min}$, video recordings of $30 \mathrm{~s}$ each were stored and later assessed again by two independent, experienced (international ultrasound diploma) and blinded sonographers. Visible bubbles were graded using the Eftedal-Brubakk-Scale [9] for visual echocardiographic assessments (

Statistics and graphs were created using R and R Studio 4.0.2 (R Core Team, 2020, www.R-project.org). Due to the exponential nature of the EB scale [10], linear approximations could be used when square root transforming the EB grade for the linear regression. A big portion of the divers were measured multiple times, linear mixed effect models [11] were used to correct the repeated measures and verify the results of the linear model. We aimed at $80 \%$ power and $p<0.05$ to detect the difference of one grade in EB scale [12].

Following informed consent from participants and ethical approval through the university ethics committee, as well as following the ethical standards of the International Journal of Sports Medicine [13], the dives were monitored but not interfered with. Depending on the

- Table 1 Eftedal-Brubakk scale [9] for inert gas bubble grading in SCUBA divers and its approximate relation to bubble numbers in semi-automatic bubble counting [10].

\begin{tabular}{|l|l|l|}
\hline $\begin{array}{l}\text { Number of } \\
\text { bubbles per } \mathbf{~ c m}^{2}\end{array}$ & $\begin{array}{l}\text { Bubble } \\
\text { Grades }\end{array}$ & $\begin{array}{l}\text { Eftedal-Brubakk (EB) scale for } \\
\text { echocardiographic bubble detection }\end{array}$ \\
\hline 0 & BG0 & No bubbles visible \\
\hline 0.05 & BG1 & Occasional bubbles \\
\hline 0.2 & BG2 & At least 1 bubble/4 cardiac cycles \\
\hline 1 & BG3 & At least 1 bubble/cardiac cycle \\
\hline 3.5 & BG4 & At least 1 bubble at every cm $^{2}$ in every view \\
\hline 10 & BG5 & Whiteout - no single bubble discrimination \\
\hline
\end{tabular}


measurement results after their dive, the participants received safety information only. The study was supported by the German Society of Diving and Hyperbaric Medicine (GTÜM e.V.) and by General Electric Healthcare's ultrasound division in Germany through material provisions.

\section{Results}

\section{Dive and individual parameters}

Of 342 dives, 101 were completed by women and 241 by men. All divers were medically fit to dive (certified by a physician according to German GTÜM guidelines). However, 72 dives were performed by divers with chronic diseases, 7 dives were done by divers smoking more than 1 pack * year, 60 by divers smoking less than 1 pack * year and 275 by non-smokers. Age and BMI distribution, as well as dive parameters, are displayed in > Fig. 1.

From a total of 342 dives, 161 dives were positive for visible bubbles in the right atrium and ventricle and also the inferior vena cava after the dive. Visible bubbles occurred especially in deep and long dives, divers over 30 years, and short surface intervals after the previous dive (residual inert gas supersaturation). Interrater reliability

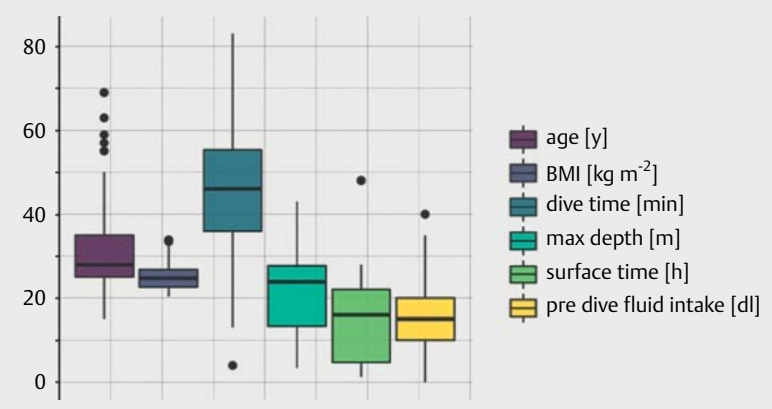

- Fig. 1 Bio-data and dive profiles of the monitored dives. Depth is always recorded as maximum depth during the dive. Dive profiles covered a broad spectrum within typical sports diving limits that were considered safe, dive computers were worn continuously, recommended safety stops and few single-step decompression stops according to commercial dive computer recommendations were followed.

- Table 2 Spearman's correlation between echocardiographically detected bubble grades and individual as well as diving parameters.

\begin{tabular}{|l|c|}
\hline Eftedal-Brubakk bubble grade 1-5 & Spearman's rho \\
\hline Maximum Depth (meters) & $0.46^{* * *}$ \\
\hline Air consumption equivalent to surface pressure $\left(\right.$ bar $\left.^{*} \mathrm{l}\right)$ & $0.41^{* * *}$ \\
\hline Age of the diver (years) & $0.25^{* * *}$ \\
\hline Dive time (minutes) & $0.23^{* * *}$ \\
\hline Decompression dive & $0.19^{* * *}$ \\
\hline Surface time before the dive (hours) & -0.12 \\
\hline $\begin{array}{l}\left(^{* * *} \mathrm{p}<0.001,{ }^{* *} \mathrm{p}<0.01,{ }^{*} \mathrm{p}<0.05, \text { adjusted } \mathrm{p} \text {-values). Surface time }\right. \\
\text { showed a significant linear correlation but } \mathrm{p}=0.05 \text { only in Spearman's } \\
\text { categorical correlation with bubble grades. }\end{array}$ \\
\hline
\end{tabular}

was 0.6 for all dives with main differences between EB grade 0 and $1(2=6.3 \%, 3=3.3 \%, 4=1.2 \%, 5=0.3 \%)$. All disagreements were reevaluated in a third video rating. Relations of dive and individual parameters to bubble grades are displayed in > Table 2.

No significant correlation was found between bubble grades and difference in blood pressure $(p=0.056)$, heart rate $(p=0.23)$ height $(p=0.63)$, weight $(p=0.84)$ and relative weight loss $(p=0.19)$, freezing during the dive (categorical, $p=0.14$ ), impedance derived body muscle, fat and water contents ( $p>0.27)$, stress and problems during the dive (categorical, $p>0.19$ ), and smoking (categorical, $p=0.54$ ). Body weight adjusted pre-dive daily fluid intake showed a borderline correlation and minimal effect size only. Male divers were found to be diving deeper ( $t$-test, $p=0.002$ ), longer ( $t$-test, $p=0.006$ ), and consumed more compressed gas ( $t$-test, $p=0.000$ ) than female divers, but there was no correlation of EB-Grade and gender $(p=0.15)$.

\section{Distribution of correlated parameters within echocardiographic bubble grade categories}

Since breathing while diving can only be done with a breathing gas pressure that is equal to the surrounding water pressure, the total air consumption in bar *I roughly combines the effects of dive time, depth and physical/psychological exertion - relevant for under water inert gas uptake - and is displayed in relation to the bubble grade detected via ultrasound in $>$ Fig. 2

\section{Multiple regression analysis of combined parameters and approximation of bubble grades}

As shown in — Table 1, the EB scale does not resemble a linear scale of bubbles per $\mathrm{cm}^{2}$, thus the variable bubbles per $\mathrm{cm}^{2}$ had to be transformed with a fourth root to achieve normal distribution of the residuals. Using multiple regression, a significant non-linear relationship between the response variable bubbles per $\mathrm{cm}^{2}$ and the variables surface time, age, maximum depth, and air consumption was found ( $\triangleright$ Table 3 ).

To avoid bias of the regression model due to the fact that the experimental design is not fully cross-sectional and most individuals were surveyed for multiple dives, the individual factor was assessed using a random effects model, with the individual (ID) as random effect. For the random effects model with the same predictors as in the linear model we found a marginal pseudo- $\mathrm{R}^{2}$ of 0.29 for the fixed effects and a conditional pseudo- $R^{2}$ of 0.37 for fixed and random effects, showing that the individual itself explained only a very small portion of the bubble grade ( $\sim 8 \%$ of the variance, ICC (intra class correlation) $=0.11$ ). Moreover, all predictors later used in the simple regression were also significant in the random-effects regression ( $p<0.05$ for age, surface time, max depth and air consumption) and therefore a simple linear approach was applicable. Furthermore, the data was subsampled multiple times into training and testing data (with a ratio of $30 \%$ and $70 \%$ ), where the linear model proved to approximate the measured EBgrade correctly.

Thus, we can use the parameters as displayed in $\vee$ Table 4 to create a practical formula that predicts the bubble grade for an individual: 

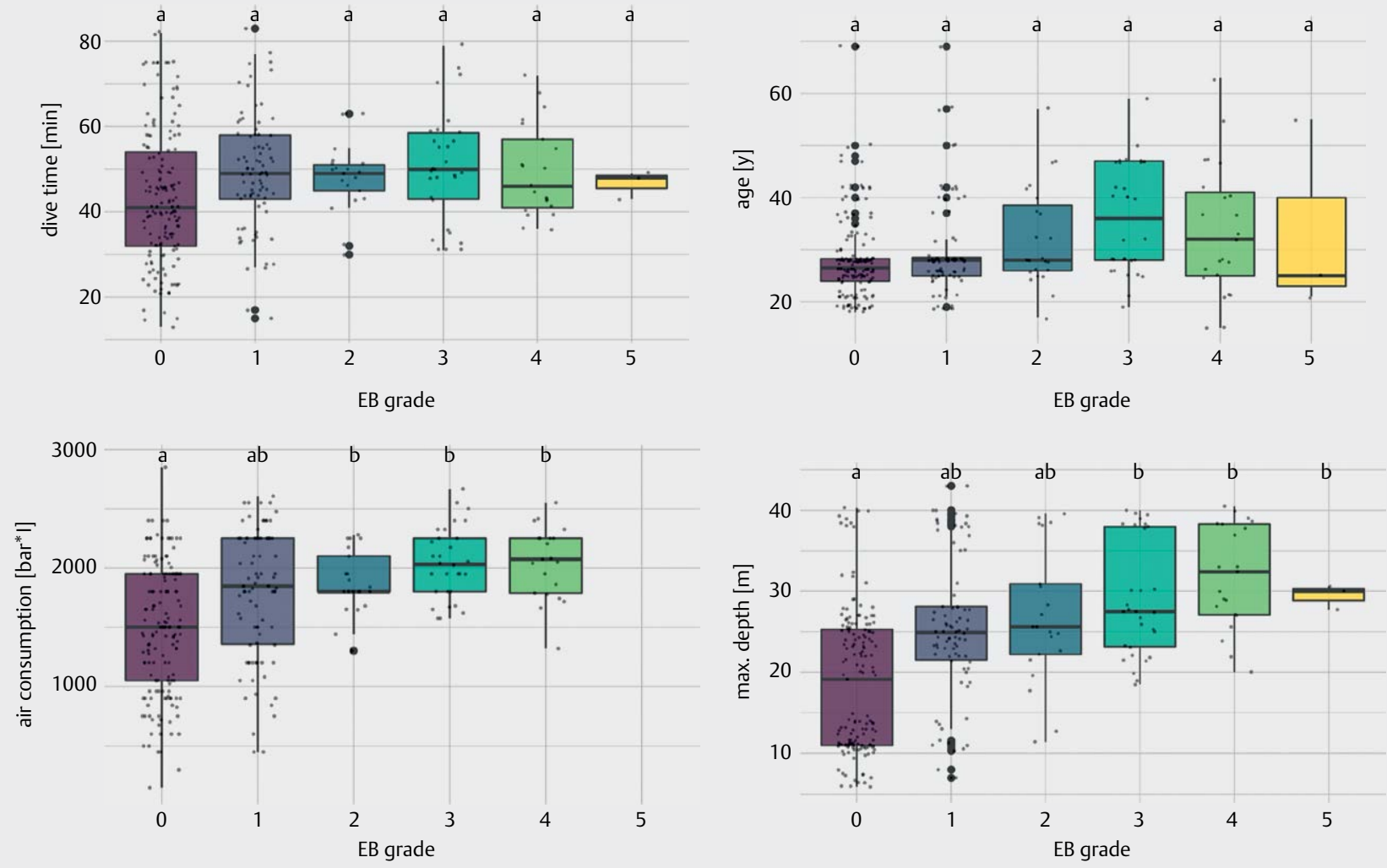

- Fig. 2 Eftedal-Brubakk bubble grades detected by echocardiography depending on depth, air consumption, age and surface time. Bubble grade 5 (whiteout) was visible after only 3 dives. Apart from fatigue in a few divers, that could be related with symptoms of a decompression incident but did not correlate with a higher bubble load, no other typical symptoms occurred. Small dots represent included data, and bold dots represent statistical outliers. Bars not sharing the same letters $(a, b)$ are significantly different from each other $(p<0.05$, Tukey HSD test for unbalanced ANOVA), bars sharing the same letter are not significantly different from each other.

- Table 3 Results of multiple regression of the combined independent parameters.

\begin{tabular}{|l|c|c|c|c|}
\hline Predictor & $\begin{array}{l}\text { Standardized } \\
\text { estimate (beta) }\end{array}$ & $\begin{array}{l}\text { t-value } \\
\text { (beta }=\mathbf{0} \text { ) }\end{array}$ & p-value & VIF \\
\hline Age (y) & 0.216 & 3.960 & 0.000 & 1.02 \\
\hline Surface time (h) & -0.1433 & -2.567 & 0.011 & 1.07 \\
\hline Max. depth (m) & 0.308 & 4.207 & 0.000 & 1.83 \\
\hline $\begin{array}{l}\text { Air consumption } \\
\text { (bar }{ }^{*} \text { I) }\end{array}$ & 0.177 & 2.477 & 0.014 & 1.76 \\
\hline
\end{tabular}

The regression was calculated with the number of bubbles per $\mathrm{cm}^{2}$. The variance inflation factor $(\mathrm{VIF}<2)$ indicates no problematic multicollinearity within this model. The dependent variable was transformed beforehand with a double square root transformation. $\mathbf{R}^{\mathbf{2}} \mathbf{= 0 . 3 0 5}$ and adjusted $R^{2}=0.294 F(4,239): 26.24$, p-value $<0.01$.

- Table 4 Formula parameters to predict EB scale grades: $R^{2}=0.3131$, Adjusted $R^{2}=0.3016 ; F(4,239)$ : 27.23, $p$-value $<0.001$ (Constant $=$ regression y-intercept).

\begin{tabular}{|l|r|l|l|}
\hline Predictor & \multicolumn{1}{|l|}{ Estimate } & Std. Error & p-value \\
\hline Constant & $-6.843^{*} 10^{-01}$ & $1.797^{*} 10^{-01}$ & 0.000 \\
\hline Age $(\mathrm{y})$ & $1.968^{*} 10^{-02}$ & $4.861^{*} 10^{-03}$ & 0.000 \\
\hline Surface time $(\mathrm{h})$ & $-6.831^{*} 10^{-03}$ & $2.494^{*} 10^{-03}$ & 0.007 \\
\hline Max. depth $(\mathrm{m})$ & $2.285^{*} 10^{-02}$ & $5.441^{*} 10^{-03}$ & 0.000 \\
\hline Air consumption $\left(\right.$ bar $\left.^{*} \mathrm{I}\right)$ & $2.302^{*} 10^{-04}$ & $8.955^{*} 10^{-05}$ & 0.011 \\
\hline
\end{tabular}

This can be used to derive a useful "field formula" as follows in order to predict Eftedal-Brubakk bubble grades after a dive from minimal dive and individual parameters:

EB bubble grade $[0-5]=(0.0196785 *$ age $[y ; 15-69]$

$-0.0068313 *$ surface time [h; $1-48]$

$+0.0228502 *$ max depth [m; 3-43]

$+0.0002302 *$ air consumption [bar * I; $150-2850])^{2}$

which can be approximated by:

EB grade $=\left(\begin{array}{l}\frac{\text { age }[\mathrm{y}]}{50}+\frac{\text { surface time }[\mathrm{h}]}{150}+ \\ \frac{\text { max depth }[\mathrm{m}]}{45}+\frac{\text { air consumption }\left[\text { bar }^{*} \mathrm{l}\right]}{50}\end{array}\right)^{2}$

Equation 1: Field formula to approximate risk of bubbling.

Due to the categorical definition of the presence or absence of bubbles and the possible underestimation of grade 0 , the intercept of -0.68 is not included into the formula ( $\triangleright$ Fig. 3, Equation 1 ). 


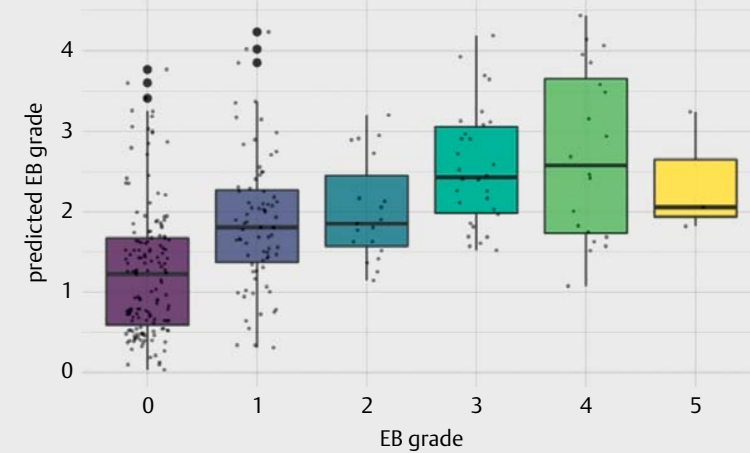

- Fig. 3 Prediction of Eftedal-Brubakk bubble grades using a formula of four variables only (age, surface time, maximum depth, and total air consumption). Small dots represent included data, and bold dots represent statistical outliers. The recording of surface time was limited to $48 \mathrm{~h}$ in our data, however, a surface time of more than $10 \mathrm{~h}$ did not reveal any difference.

\section{Discussion}

\section{Parameters correlating with bubble grade}

In the past decades, there has been abundant research on safe diving parameters and decompression algorithms from an ex-ante view in order to avoid decompression stress and finally a decompression incident from critical inert gas supersaturation and major bubbling [14]. More recently, individual parameters causing a diving accident despite following empiric and calculated decompression rules were focused on from an ex-post view $[15,16]$. Today, ultrasound examinations after a dive, which are still done mainly by medical experts for research, add valuable information on individual decompression stress without symptoms of a diving accident $[8,17-20]$ and help to initiate appropriate measures like extending surface intervals, breathing oxygen or increasing fluid intake to avoid a diving accident. Within a broad interindividual cohort and a variety of sports diving profiles within standard commercial dive computer limits, our study revealed bubbling in $47 \%$ of all dives and of all grades including whiteout. Known factors related to bubbling are diving exposure (depth, dive time, reduced surface interval), as well as BMI, age [7] and also diminished fluid status. Our study confirms the influence of diving exposure parameters. From individual factors, fat is known to increase inert gas storage capacity and is not related to higher bubble grades [16] right after the dive, as also confirmed by our data. Conflicting results [7] may be related to BMI-dependent impairment of physical condition and thus higher exertion and inert gas uptake. A repeated bubble grading was not carried out in this pilot study since precisely determined individual bubble peak curves were not relevant. The aim was instead to relate a broad spectrum of diving and individual parameters to post-dive bubbling at the same time interval of measurement that has already been found to be within the typical peak bubble time after sports SCUBA dives. The effect of small timely differences in bubble occurrence around the typical and previously published time interval of peak bubbling was expected to be lower than the effect of non-linear categorical bubble grading for a diver-oriented level of accuracy in detecting a relevant bubble load.

\section{Relevant parameters for bubble grade approximation}

Most impressive is the strong correlation and moderate effect size of air consumption and age of the divers. Together with maximum depth and surface time, it was possible to find a formula predicting post-dive bubbling reasonably well. Although depth is related to air consumption of a dive, as is also dive time, depth still contributes a significant independent factor to bubble grade calculation more than dive time. This is due to the same air consumption that can occur in long shallow dives without relevant inert gas uptake compared to shorter deep dives with relevant inert gas saturation. Surface pressure equivalent air consumption in bar * I appeared to be a more suitable model mainly for diving exposure intensity and - to a smaller extent - individual metabolic activity than just maximum depth or diving duration. Some divers in our study seemed to be more prone to bubbling than others. However, after comparing dive profiles, we recognized a higher specific air consumption of these individuals. For example, male divers were diving deeper, longer, and consumed more air than female divers on average, yet there is no association with gender and EB grade in our data. All this suggests that the person-specific likelihood of an increased EB grade may be also a product of exposure intensity suitably shown by personal air consumption with further contributing individual factors such as age.

Several studies have shown that some individuals are more susceptible to DCI than others [15, 21, 22]. However, the individual effect was, in fact, measured in our model ICC (intra-class correlation coefficient $)=0.11$ and explained only about $8 \%$ of the variance of the EB grade (Pseudo- $\mathrm{R}^{2}$ difference). The fixed effect parameters (depth, air consumption, surface time, age) explained the same variance in the model with the random effect (the individual) as in the model ignoring the random effect, we therefore proceeded with the latter, as the difference of this individual deviation to the estimated risk proved to be minor. The formula (Equation 1) can be seen as independent of this individual effect. Due to the high variability of the divers and diving profile a high variance was induced into our estimation, which can be expected in a field measurement.

\section{Limitations and potential for optimization and interpretation}

We tried to find a simple, generalizable relation between dive and individual parameters in order to account for the additional information on relevant decompression stress, which can only be provided by a professional post-dive echocardiography. It was not possible for us to estimate whether individuals are susceptible differently to bubbling, as we did not have a standardized diving procedure and individual differences could also result from more risky diving behavior (i. e. there is a difference between men and women in the EB grade, but men also tend to dive deeper than women). With a standardized post-dive measurement, the individual and the dive parameters, as well as the resulting decompression stress, were our variable factors within the framework of standard sports diving profiles.

A shortcoming in our study is the categorical Eftedal-Brubakk scale of echo-bubble grading that we tried to accomplish with our 
non-linear formula. Especially in extreme bubble grades 0 and 5 , the fit seems to be not optimal, yet the model can identify increased risks. Nevertheless, considering that a visible bubble grade 0 is challenging to judge, as a few bubbles that would define grade 1 or even grade 2 can be easily missed during approximately one minute of ultrasound scanning, the slight overestimation of our formula in this category seems to be quite realistic. On the other side of the scale, we had only three dives that showed a bubble grade 5 (whiteout) after the ascent. Furthermore, it is a big step between grade 4 (>1 bubble $\mathrm{cm}^{-2}$ ) and whiteout without visible bubble discrimination. Therefore, it is challenging to predict grade 5 with our data reliably.

In order to find a better prediction of decompression stress with a possible linear relation, it seems necessary to leave the categorized scale towards a counted number of high-intensity transient signals, e. g., in ultrasound recordings of the inferior vena cava over time and semiautomatic counting [23] of visible or acoustic bubble signals. Further, timely variability of individual peak bubbling can be missed with our standardized, but single measurement approach. However, the relevant diagnostic information is not impaired by slight under- or overestimation of the bubble load. Other rough - more or less categorical - data like maximum depth (neglecting depth-time integral as much more fundamental factor for inert gas uptake), air consumption (neglecting air used for buoyancy control and the primary influence of depth) and EB bubble grading influence diagnostic accuracy significantly. Despite these biases, we were able to show a significant relation of a diver-oriented combination of simple individual and dive-related parameters to approximate relevant bubble load.

\section{Conclusion}

It is possible to predict echocardiographically-derived bubble grading after a dive and therefore to generate information on decompression stress from inert gas bubbling in a categorical manner using a calculation based on easily accessible dive and individual parameters. Validation and adjustment with a large number of dives and a correlation to a more linear bubble grading with automatic integration, especially in dive computers with tank pressure sensors, could potentially contribute to individual diving safety.

\section{Acknowledgements}

We highly acknowledge technical support and data collection by Denise Preuss ${ }^{2}$, Anne Muench ${ }^{4}$.

\section{Funding}

Open Access Funding by the Publication Fund of the TU Dresden.

2 Emergency Department, Kreiskrankenhaus Freiberg gGmbH, Freiberg, Germany.

4 Scientific Diving Center, TU Bergakademie Freiberg University, Freiberg, Germany.

\section{Conflict of Interest}

The authors declare that they have no conflict of interest.

\section{References}

[1] Arieli R, Marmur A. A biophysical vascular bubble model for devising decompression procedures. Physiol Rep 2017; 5(6): e13191

[2] Carturan D, Boussuges A, Vanuxem P et al. Ascent rate, age, maximal oxygen uptake, adiposity, and circulating venous bubbles after diving. J Appl Physiol (1985) 2002; 93: 1349-1356

[3] Cialoni D, Brizzolari A, Samaja M et al. Altered venous blood nitric oxide levels at depth and related bubble formation during scuba diving. Front Physiol 2019; 10: 57

[4] Blogg SL, Gennser M, Møllerløkken A et al. Ultrasound detection of vascular decompression bubbles: The influence of new technology and considerations on bubble load. Diving Hyperb Med 2014; 44: 35-44

[5] Eftedal O, Brubakk AO. Detecting intravascular gas bubbles in ultrasonic images. Med Biol Eng Comput 1993; 31: 627-633

[6] Eftedal OS, Lydersen S, Brubakk AO. The relationship between venous gas bubbles and adverse effects of decompression after air dives. Undersea Hyperb Med 2007; 34: 99-105

[7] Dive risk factors, gas bubble formation, and decompression illness in recreational SCUBA diving: Analysis of DAN Europe DSL Data Base. Front Psychol 2017; 8: 1587

[8] Møllerløkken A, Blogg SL, Doolette D] et al. Consensus guidelines for the use of ultrasound for diving research. Diving Hyperb Med 2016; 46: $26-32$

[9] Eftedal O, Brubakk AO. Agreement between trained and untrained observers in grading intravascular bubble signals in ultrasonic images. Undersea Hyperb Med 1997; 24: 293-299

[10] Eftedal OS. Ultrasonic Detection of Decompression Induced Vascular Microbubbles. Thesis for the degree of doctor philosophiae, Norwegian University of Science and Technology, Faculty of Medicine 2007; ISBN 978-82-471-3697-3

[11] Bates D, Mächler M, Bolker B et al. Fitting linear mixed-effects models using Ime4. J Stat Softw 2015; 67: 1-48

[12] Doolette DJ, Gault KA, Gutvik CR. Sample size requirement for comparison of decompression outcomes using ultrasonically detected venous gas emboli (VGE): Power calculations using Monte Carlo resampling from real data. Diving Hyperb Med 2014; 44: 14-19

[13] Harriss DJ, MacSween A, Atkinson G. Ethical standards in sport and exercise science research: 2020 update. Int J Sports Med 2019; 40: 813-817

[14] Hugon J. Decompression models: Review, relevance and validation capabilities. Undersea Hyperb Med 2014; 41: 531-556

[15] Lambrechts K, Pontier J-M, Mazur A et al. Effect of decompressioninduced bubble formation on highly trained divers microvascular function. Physiol Rep 2013; 1: e00142

[16] Schellart NAM, van Rees Vellinga TP, van Dijk Fj et al. Doppler bubble grades after diving and relevance of body fat. Aviat Space Environ Med 2012; 83: 951-957

[17] Spencer MP, Johanson DC. Investigation of New Principles for Human Decompression Schedules Using Doppler Ultrasonic Blood Bubble Detection. Defense Technical Information Center USA. Technical rept. no. 3, 1974; 1967-1974

[18] Kisman K, Masurel G, LaGrue D et al. Evaluation of the quality of decompression using ultrasound bubble detection. Méd Aéro Spat Méd Sub Hyp 1978; 67: 293-297 
[19] Kisman K, Masurel G. Method for Evaluating Circulating Bubbles Detected by Means of the Doppler Ultrasonic Method Using the 'K.M. Code'. Toulon, France: Centre d'Etudes et Recherches Techniques Sous-Marines 1983

[20] Nishi RY, Kisman KE, Eatock BC et al. Assessment of decompression profiles and divers by Doppler ultrasonic monitoring. In: Bachrach AJ, Matzen MM, Eds. Underwater Physiology VII. Proceedings of the Seventh Symposium on Underwater Physiology. Bethesda, MD: Undersea Medical Society. 1981: 717-727

[21] Arieli R. Nanobubbles Form at active hydrophobic spots on the luminal aspect of blood vessels: Consequences for decompression illness in diving and possible implications for autoimmune disease-an overview. Front Physiol 2017; 8: 591
[22] Cialoni D, Pieri M, Balestra C et al. Flying after diving: Should recommendations be reviewed? In-flight echocardiographic study in bubble-prone and bubble-resistant divers. Diving Hyperb Med 2015; 45: $10-15$

[23] Eftedal O, Brubakk AO, Nishi RY. Ultrasonic evaluation of decompression: The relationship between bubble grades and bubble numbers. Undersea Hyperb Med 1998; 25suppl: 1-60, (abstract 35-36) 